\title{
Pain, Function, and Satisfaction After Total Knee Arthroplasty, with or Without Bariatric Surgery
}

\author{
Perna Ighani Arani ${ }^{1,2}\left(\mathbb{D} \cdot\right.$ Per Wretenberg ${ }^{1,2} \cdot$ Johan Ottosson ${ }^{2,3,4} \cdot$ Annette W-Dahl $^{5,6}$
}

Received: 30 August 2021 / Revised: 13 January 2022 / Accepted: 14 January 2022 / Published online: 27 January 2022

(c) The Author(s) 2022

\begin{abstract}
Background The impact of obesity on patient-reported outcome (PRO) after total knee arthroplasty (TKA) surgery has demonstrated varying results. We evaluated knee pain, Activity in Daily Life function (ADL), and satisfaction after TKA surgery in patients with and without prior bariatric surgery (BS).

Methods Scandinavian Obesity Surgery Registry (SOReg) and the Swedish Knee Arthroplasty Register (SKAR) were used to identify patients operated on with primary TKA for osteoarthritis (OA) between 2009 and 2019 that had a BS within 2 years before the TKA (BS group). These patients were compared to patients with TKA without prior BS (no BS group). The patients filled in the Knee injury and Osteoarthritis Outcome Score (KOOS) preoperatively and one year postoperatively as well as satisfaction with the surgery one year postoperatively. Multiple linear regression analysis was used to evaluate 1-year postoperative KOOS pain and ADL function between the 2 groups. Adjustments were made for sex, age, and preoperative KOOS pain and ADL function respectively.

Results Forty-four patients were included in the BS group and 3,525 patients in the no BS group. We found no statistically or clinically significant difference in one-year postoperative KOOS pain and ADL function between the BS group and the no BS group. The majority of the patients in both groups were classified as satisfied or very satisfied one year postoperatively to the TKA.

Conclusions Our results indicate that patients without BS prior to the TKA gain similar 1-year outcome in pain, ADL function and satisfaction as patients with prior BS.
\end{abstract}

Keywords Obesity $\cdot$ Bariatric surgery $\cdot$ Osteoarthritis $\cdot$ Total knee arthroplasty $\cdot$ Patient-reported outcome

\section{Introduction}

Key Points • The results indicate similar pain and ADL function one year after TKA with or without prior BS.

- We found an improvement in ADL and pain one year postTKA regardless of prior BS.

- The result indicates similar satisfaction with the TKA regardless of prior BS.

Perna Ighani Arani

perna.arani@gmail.com

Per Wretenberg

per.wretenberg@regionorebrolan.se

Johan Ottosson

johan.ottosson@regionorebrolan.se

Annette W-Dahl

annette.w-dahl@med.lu.se

1 Department of Orthopedics, Örebro University Hospital, Örebro, Sweden
Obesity in the society is a medical challenge that continues to increase among the population in Europe [1]. Previous studies have shown that obesity has a negative impact on complications and mortality after total knee arthroplasty (TKA) [2-4]. Studies evaluating the impact of obesity on

2 Faculty of Medicine and Health, School of Medical Sciences, Örebro University, 70281 Örebro, Sweden

3 Department of Surgery, Örebro University Hospital, Örebro, Sweden

4 Scandinavian Obesity Surgery Registry, Örebro, Sweden

5 Department of Clinical Sciences Lund, Faculty of Medicine, Lund University, 22100 OrthopedicsLund, Sweden

6 The Swedish Knee Arthroplasty Register, Lund, Sweden 
patient-reported outcome (PRO), such as pain and knee function, have demonstrated varying results [5, 6]. Our previous findings did not indicate that having a BS prior to TKA was associated with lower risk of revision [7]. Over time, the proportion of patients with obesity undergoing TKA has steadily been increasing [8]. We have recently published a study demonstrating that patients with BS prior to TKA had an increased risk of revision due to infection, compared to patients without prior BS. The control group consisted of patients within the same BMI range before their TKA [7]. Consequently, it is of clinical interest to determine if these patients were less satisfied regarding pain and ADL function one year after TKA.

Bariatric surgery has been demonstrated to be an effective method of reaching significant long-term weight loss in comparison with nonsurgical interventions in patients with obesity [9]. There are no previous studies examining the association of BS on PROs postoperatively to TKA. Therefore, the aim of this study was to evaluate pain and ADL function and satisfaction one year postoperatively in patients having BS before the TKA and compare them to TKA patients without BS.

\section{Patients and Methods}

We used a subset of a cohort including patients who underwent primary gastric bypass or sleeve gastrectomy (BS) in 2007-2019 identified from the Scandinavian Obesity Surgery Register (SOReg) and patients having primary TKA for OA in 2009-2019 within 2 years after their BS, and patients without prior BS from the Swedish Knee Arthroplasty Register (SKAR). As previously described, we used the BMI prior to the TKA in the BS group, and selected the no BS patients within the same BMI interval (range 16.9-50) and age interval (41.9-67 years) as the BS group [7]. The SOReg was established in 2007 and the SKAR in 1975. The two registers have high completeness and correctness, validated through the national patient register $[10,11]$. The subset consisted of patients having BS surgery and TKA surgery in the Region of Skåne, in the southern part of Sweden. The PROs (preoperatively and one year postoperatively) were obtained from the SKAR PROM project. The SKAR PROM project is voluntary and started in 2008 with one hospital and has extended to include 39 hospitals in 2020. Each of the 21 regions in Sweden has responsibility for the health care provided to the inhabitants. We included only the Region of Skåne as all hospitals in the region report PRO measures to the SKAR and BS is performed in the region as well. BMI, age, sex, and ASA classification were obtained from the SKAR.

In patients who had BS before the TKA (BS group), we excluded the $2^{\text {nd }}$ knee if both knees had been operated after the BS. In patients without prior BS to TKA (no BS group), we excluded the patients that were found in the SOReg. The $2^{\text {nd }}$ TKA were excluded in those who underwent staged bilateral surgery, and in patients who underwent simultaneous bilateral TKA, the left knee was excluded (Fig. 1). Only patients with complete PROs were included in the study (Fig. 1).

\section{KOOS}

The Knee injury and Osteoarthritis Outcome Score (KOOS) is a patient-reported outcome measure (PROM), which assess the patient's own experience about their knee and associated issues. It consists of 42 questions divided in 5 subscales: Pain, Symptoms, Activity in Daily Life function (ADL), Sport and Recreation Function (Sport/rec), and Quality of Life (QoL). Scores are transformed to a 0-100 scale, with 0 indicating extreme knee problems and 100 indicating no knee problems [12]. A score of $\geq 8$ points in the KOOS was considered a clinically relevant difference for statistically significant results [13].

\section{OMERACT-OARSI Responder}

The Outcome Measures in Rheumatology (OMERACT) committee and The Osteoarthritis Research Society International (OARSI) Standing Committee for Clinical Trials Response Criteria Initiative have developed OMERACTOARSI criteria, which are outcome measures for osteoarthritis that are based on the combination of absolute and relative changes in Western Ontario and McMaster Universities Osteoarthritis Index (WOMAC) pain, functional impairment, and total score one year postoperatively [14].

The KOOS was converted to WOMAC before classifying each patient by the OMERACT-OARSI criteria into high, low, or no responders one year postoperatively [10]. High responder is defined as a patient with an improvement of $\geq 20$ points, which should also correspond to $\geq 50 \%$ improvement in pain or function, compared to preoperative WOMAC score. Low responder is defined as improvement of $\geq 10$ points also corresponding to $\geq 20 \%$ in two of the WOMAC pain, function, or total score.

\section{Patient Satisfaction}

The patients' satisfaction with the TKA surgery one year postoperatively was assessed by using a 0-100 Visual Analogue Scale, where 0 is the high satisfaction and 100 is no satisfaction. Depending on the score given, the patients were classified into 5 groups; very satisfied (0-20), satisfied (21-40), moderately satisfied (41-60), not satisfied (61-80), and very unsatisfied (81-100). 


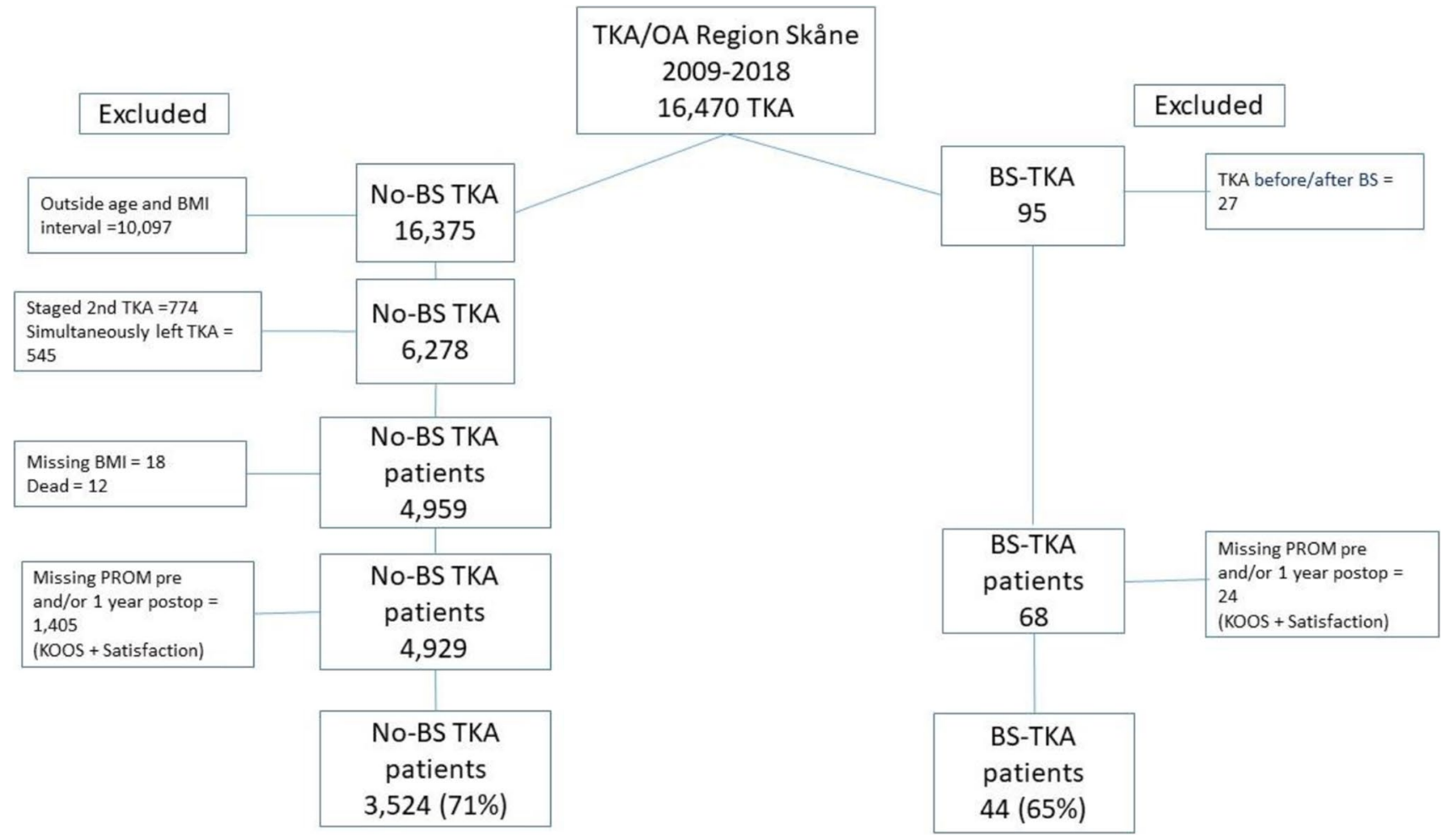

Fig. 1 Flow-chart of the study population

\section{Statistics}

Patient characteristics were presented as means with standard deviations (SD) or numbers and percent when appropriate. The KOOS subscales were presented as means with $95 \%$ confidence intervals (CI).

Multiple linear regression analysis was performed to evaluate one-year postoperative pain and ADL function between the 2 groups. We adjusted for sex, age and BS or not, and preoperative KOOS pain and ADL function respectively. BMI was not included as a confounder since the difference between the cohorts was only one unit.

Statistical analyses were carried out using Stata version 15 (StataCorp, College Station, TX, USA).

\section{Ethics, Funding, and Potential Conflicts of Interest}

The study was conducted according to the declaration of Helsinki [15], and was also waived by the Swedish Ethical Review authority (2017/466). Informed consent does not apply. The authors have no conflicts of interest to report.

\section{Results}

Forty-four patients operated on with bariatric surgery prior to the TKA for OA were included in the BS group and 3,524 patients without BS were included in the no BS group. The BS group was younger (mean age: 56 vs 60 years) and had a higher proportion of females ( $86 \%$ vs $57 \%$ ) compared to the no BS group. The majority of patients had an ASA score of 2 in BS and no BS group (70\% and $62 \%$ respectively) (Table 1 ). The BS group had a mean reduction of 14.2 (SD 3.3) in BMI after $\mathrm{BS}$, and a mean total weight loss of $33.6 \%$ (SD 12\%). The mean time between BS and TKA was 1.1 years (SD 0.43).

The BS group reported statistically significant worse KOOS scores 1-year postoperatively for pain, but not for ADL (Pain: 71 (CI 64-77), ADL: 75 (CI 69-82)) than the no BS group (Pain: 78 (CI 77-79), ADL: 78 (CI 78-79)), but without clinically relevant differences (Table 2). When adjusting for sex, age and BS or not, and preoperative KOOS pain and ADL function, respectively, we found no statistically significant difference between the groups (Pain: 4.2 (CI 1.4-9.7), ADL: 1.0 (CI 4.4-6.4)) (Table 3). The majority of the BS and the no BS group were high responder according to the OMERACT-OARSI criteria and were classified into satisfied or very satisfied one year postoperatively to TKA (Table 2).

\section{Discussion}

We found no clinically relevant difference in one-year postoperative pain and ADL-function nor satisfaction and OMERACT-OARSI responder between patients with BS 
Table 1 Patient characteristics and preoperative KOOS for included and excluded/missing TKA patients with (BS) or without prior BS (no BS)

\begin{tabular}{llll}
\hline & BS & No BS & $p$ values \\
& $N=44$ & $N=3,524$ & \\
\hline $\begin{array}{l}\text { Age in years, mean (SD) } \\
\text { Sex, } n \text { (\%) }\end{array}$ & $55.9(5.7)$ & $60.4(5)$ & $<0.001$ \\
Female & 38 & $2,024(57.4)$ & \\
Male & 6 & $1,500(42.6)$ & \\
ASA classification, $n(\%)$ & & & $<0.001$ \\
1 & 7 & $1,028(29.2)$ & \\
2 & 31 & $2,175(61.8)$ & \\
$\geq 3$ & 6 & $318(9.0)$ & \\
BMI, mean (SD) & $30.6(3.7)$ & $29.5(4.4)$ & 0.053 \\
Preoperative KOOS score, & mean (CI) & & \\
Pain & $32(29-36)$ & $37(37-38)$ & 0.012 \\
ADL & $43(39-47)$ & $45(44-46)$ & 0.409 \\
Symptom & $37(32-42)$ & $42(41-43)$ & 0.050 \\
Sport/rec & $6(3-9)$ & $10(9-10)$ & 0.008 \\
QOL & $19(16-23)$ & $20(20-20)$ & 0.767 \\
\hline
\end{tabular}

$S D$, standard deviation; $C I, 95 \%$ confidence interval; $A S A$, American Society of Anaesthesiologists

prior to the TKA and those without BS. To our knowledge, this is the first study investigating the association between $\mathrm{BS}$ or no BS prior to TKA and PROs.

However, our study has limitations. We included relatively few patients in the BS group indicated by the relatively wide CIs, which mean that we may not be able to show differences that may exist. The rationale to include

Table 2 One-year postoperative KOOS, OMERACT-OARSI responders, and satisfaction with the surgery in TKA patients with prior BS or without prior BS

\begin{tabular}{llll}
\hline & BS & No BS & $p$ value \\
& $N=44$ & $N=3,524$ & \\
\hline Postoperative KOOS score, mean (CI) & & \\
Pain & $71(64-77)$ & $78(77-79)$ & 0.033 \\
ADL & $75(69-82)$ & $78(78-79)$ & 0.337 \\
Symptom & $68(62-73)$ & $72(72-73)$ & 0.100 \\
Sport/rec & $32(24-40)$ & $35(34-36)$ & 0.454 \\
QOL & $59(52-66)$ & $60(60-61)$ & 0.623 \\
OMERACT-OARSI responder, $n(\%)$ & & \\
High responder & 36 & $2,820(80,0)$ & \\
Low responder & 3 & $293(8,3)$ & \\
No responder & 5 & $411(11,7)$ & \\
Satisfaction, $n$ (\%) & & & \\
Very satisfied/satisfied & 37 & $2,911(82.6)$ & \\
Moderate satisfied & 4 & $368(10.4)$ & \\
Very unsatisfied/not satisfied & 3 & $245(7.0)$ & \\
\hline
\end{tabular}

CI $95 \%$ confidence interval
Table 3 The relationship between BS or no BS prior to TKA and one-year postoperative KOOS pain and ADL function respectively

\begin{tabular}{ll}
\hline 1-year postoperative KOOSpain* & $\begin{array}{l}\text { 1-year postoperative KOOS } \\
\text { ADL* }\end{array}$ \\
Coefficient $(95 \% \mathrm{CI})$ & Coefficient $(95 \% \mathrm{CI})$ \\
BS $(N=44)$ reference & Reference \\
No BS $(N=2,524) 4.2(-1.4$ to 9.7$)$ & $1.0(-4.4$ to 6.4$) p=0.717$ \\
$p=0.145$ & \\
\hline
\end{tabular}

*Adjusted for age, sex, and preoperative KOOS pain and ADL function respectively

only one region was that the SKAR PROM project has PRO measures from the Region of Skåne since 2008, and BS is also performed in the region. Furthermore, it is an observational study with limited variables included. With the relatively low number of TKA patients with prior BS, we limited our analysis and used the available variables relevant as potential confounders.

However, the data from the SOReg and the SKAR is prospectively collected and both registers are known for having a high completeness and quality $[10,16]$.

The mean age of patients undergoing BS in Sweden is slightly above 40 years, and a majority of these patients are women [16]. This is in line with the cohort included in the current study. In order to make the groups more comparable, we selected the patients in the no BS group within the same age interval as those in the BS group. When filtering the selection of the no BS group regarding BMI, we used the BS groups BMI prior to the TKA. We did not include BMI in the regression analysis as the difference in BMI was only one unit. We decided not to adjust for the ASA classification as BMI interferes with comorbidity [17], and BMI is in addition a factor in deciding the ASA classification. A majority of the patients in both cohorts were classified to ASA 2, which demonstrate that most of the patients were relatively healthy or suffered from a mild systemic disease. We did a sensitivity analysis were we included ASA as confounder; however it did not change the results. The cohort includes the two most common bariatric surgeries in the BS group; gastric bypass and sleeve gastrectomy (95\% and 5\% respectively). Due to low number of patients in the BS group, no subgroup analysis was done.

A majority of the patients in both groups were high responder and "very satisfied" or "satisfied" one year postoperatively to TKA. Overgaard et al. (2019) compared PROs in different BMI categories and showed similar satisfaction rates among the BMI categories. Compared to mean values, which can be affected by outliers and hide both good and bad outcome, the OMERACT OARSI responder presents a clearer picture of the patients' improvement from given criteria. 
Obesity has been associated to worse outcomes in patients undergoing TKA, primarily when it comes to the risk of revision [18]. During the past decades, the proportion of patients with obesity undergoing primary TKA has increased in the USA, resulting in a higher incidence of revision surgeries. However, this has not been the case in Sweden where the proportion of patients with $B M I \geq 35(9.3 \%, 2019)$ having a knee arthroplasty has slightly decreased during the last decade $[10,19]$. An effective method of achieving long term weight loss is bariatric surgery [9]. Consequently, BS may reduce the risk of revision for patients having a subsequent TKA. However, in a previously study, we found the risk of revision for any reasons and for infection not to be reduced in patients having a TKA due to osteoarthritis within 2 years after BS compared to those without BS [7].

Studies on PROs after TKA in patients with obesity have shown varying results [5, 6]. Overgaard et al. (2019) evaluated the KOOS one year postoperatively to TKA and found no clinically relevant difference in pain or ADL function between different BMI groups in over 3,000 patients. On the other hand, Xu et al. (2018) showed that the patients with obesity had statistically significant poorer Oxford Knee Scores 10 years after TKA surgery when compared to those without obesity in 126 patients. However, the patients being 10 years older in the follow up may influence the outcome.

It has been postulated that weight loss improves the outcomes after TKA; both dietary and surgical methods have been proposed and tested [20-22]. In a randomized pilot trial on 40 patients with $\mathrm{BMI} \geq 30$ who were undergoing a total hip and knee replacement, the effects of a dietetic intervention were compared to usual care. An association between maintained or lost weight and better outcomes in pain and activity was demonstrated compared to patients who had gained weight one year after the intervention. However, when comparing the intervention group to the control group, they found no difference in pain, function, or activity levels [20]. Keeney et al. (2019) assessed physical function improvement in 203 morbidly obese patients, where the patients were divided into groups depending on the weight reduction $(5,10$, and 20 pounds of reduction in weight) and found no differences in physical function outcome between the groups [21]. Furthermore, Liljenso et al. (2019) investigated the effect of 8-week low energy diet on 76 TKA patients in a randomized control trial. Despite the fact that patients in the diet group maintained their weight loss one year after surgery, they found no statistically significant difference in either quality of life or knee function. However, the mean reduction was only 3.2 BMI units in the intervention group [22]. The weight reduction in these studies is much lower in the intervention groups compared to the expected weight loss after BS.

A systemic review on the effects of BS on knee complaints in patients with morbid obesity found that patients with morbid obesity undergoing BS with subsequent marked weight loss are likely to improve knee pain, physical function and stiffness. [23]. In addition, Parvizi et al. (2000) found that the Knee Society Score improved significantly 2-6 years after TKA in 7 patients with prior BS. [24]. The abovementioned studies included relatively few patients which may influence the possibility to find differences that may exist.

Some of the results mentioned are in line with our results showing no relevant difference in knee pain or function between the groups receiving intervention for weight loss and those without interventions. However, none of the discussed studies had the same objective, nor patient groups as our study, and they included as well relatively few patients. The mentioned studies also differ from our study in their objectives. In this current study, we have analyzed how the BS in itself affects PROM, and not the weight loss, in comparison to the mentioned studies.

\section{Conclusion}

Our study may indicate that patients having BS prior to their TKA have similar one year outcome regarding their pain and ADL function as compared to patients within the same age and BMI interval without BS. However, our BS group consisted of relatively few patients and future studies with larger sample size are needed.

Acknowledgements We would like to thank all the contact surgeons and associated staff at the Swedish hospitals for their dedicated registration work over the years.

Author Contribution PW, AWD, PIA and JO conceived and designed the study. AWD performed the analysis. PIA and AWD wrote the initial draft. All authors contributed to the interpretation of the data and to revision of the manuscript.

Funding Open access funding provided by Örebro University.

\section{Declarations}

Ethics Approval The study was conducted according to the declaration of Helsinki, and was also waived by the Swedish Ethical Review authority (2017/466).

Informed Consent Informed consent does not apply.

Competing Interests The authors declare no competing interests.

Open Access This article is licensed under a Creative Commons Attribution 4.0 International License, which permits use, sharing, adaptation, distribution and reproduction in any medium or format, as long as you give appropriate credit to the original author(s) and the source, provide a link to the Creative Commons licence, and indicate if changes were made. The images or other third party material in this article are 
included in the article's Creative Commons licence, unless indicated otherwise in a credit line to the material. If material is not included in the article's Creative Commons licence and your intended use is not permitted by statutory regulation or exceeds the permitted use, you will need to obtain permission directly from the copyright holder. To view a copy of this licence, visit http://creativecommons.org/licenses/by/4.0/.

\section{References}

1. Krzysztoszek J, Laudanska-Krzeminska I, Bronikowski M. Assessment of epidemiological obesity among adults in EU countries. Ann Agric Env Med. 2019;26:341-9.

2. Kerkhoffs GM, Servien E, Dunn W, Dahm D, Bramer JA, Haverkamp D. The influence of obesity on the complication rate and outcome of total knee arthroplasty: a meta-analysis and systematic literature review. J Bone Jt Surg Am. 2012;94:1839-44.

3. Tohidi M, Brogly SB, Lajkosz K, Grant HJ, VanDenKerkhof EG, Campbell AR. Ten-year mortality and revision after total knee arthroplasty in morbidly obese patients. J Arthroplasty. 2018;33:2518-23.

4. Electricwala AJ, Jethanandani RG, Narkbunnam R, Huddleston JI, Maloney WJ, Goodman SB, et al. Elevated body mass index is associated with early total knee revision for infection. J Arthroplasty. 2017;32:252-5.

5. Xu S, Chen JY, Lo NN, Chia SL, Tay DKJ, Pang HN, et al. The influence of obesity on functional outcome and quality of life after total knee arthroplasty: a ten-year follow-up study. Bone Jt J. 2018;100-B:579-83.

6. Overgaard A, Lidgren L, Sundberg M, Robertsson O, W-Dahl A. Patient-reported 1-year outcome not affected by body mass index in 3,327 total knee arthroplasty patients. Acta Orthop. 2019;90:360-5.

7. Ighani Arani P, Wretenberg P, Ottosson J, Robertsson O, W-Dahl A. Bariatric surgery prior to total knee arthroplasty is not associated with lower risk of revision: a register-based study of 441 patients. Acta Orthop. 2020;1-5.

8. Odum SM, Springer BD, Dennos AC, Fehring TK. National obesity trends in total knee arthroplasty. J Arthroplasty. 2013;28:148-51

9. Sjostrom L. Review of the key results from the Swedish Obese Subjects (SOS) trial - a prospective controlled intervention study of bariatric surgery. J Intern Med. 2013;273:219-34.

10. SKAR. SKAR. The Swedish knee arthroplasty register-annual report 2020. 2020.

11. Sundbom M, Naslund I, Naslund E, Ottosson J. High acquisition rate and internal validity in the Scandinavian Obesity Surgery Registry. Surg Obes Relat Dis. 2021;17:606-14.

12. KOOS. KOOS. Knee injury and Osteoarthritis Outcome Score. 2021.
13. Roos EM, Lohmander LS. The Knee injury and Osteoarthritis Outcome Score (KOOS): from joint injury to osteoarthritis. Health Qual Life Outcomes. 2003;1:64.

14. Pham T, van der Heijde D, Altman RD, Anderson JJ, Bellamy N, Hochberg M, et al. OMERACT-OARSI initiative: Osteoarthritis Research Society International set of responder criteria for osteoarthritis clinical trials revisited. Osteoarthritis Cartilage. 2004;12:389-99.

15. World Medical Association. World Medical Association Declaration of Helsinki: ethical principles for medical research involving human subjects. JAMA. 2013;310:2191-4.

16. SOReg. SOReg. The Scandinavian Obesity Surgery Registry. Annual report 2018, part 3/3. 2018.

17. Owens WD, Felts JA, Spitznagel EL. ASA physical status classifications: a study of consistency of ratings. Anesthesiology. 1978;49:239-43.

18. Boyce L, Prasad A, Barrett M, Dawson-Bowling S, Millington S, Hanna SA, et al. The outcomes of total knee arthroplasty in morbidly obese patients: a systematic review of the literature. Arch Orthop Trauma Surg. 2019;139:553-60.

19. Odum SM, Van Doren BA, Springer BD. National obesity trends in revision total knee arthroplasty. J Arthroplasty. 2016;31:136-9.

20. Gandler N, Simmance N, Keenan J, Choong PF, Dowsey MM. A pilot study investigating dietetic weight loss interventions and 12 month functional outcomes of patients undergoing total joint replacement. Obes Res Clin Pr. 2016;10:220-3.

21. Keeney BJ, Austin DC, Jevsevar DS. Preoperative weight loss for morbidly obese patients undergoing total knee arthroplasty: determining the necessary amount. J Bone Jt Surg Am. 2019; 101:1440-50.

22. Liljensoe A, Laursen JO, Bliddal H, Soballe K, Mechlenburg I. Weight loss intervention before total knee replacement: a 12-month randomized controlled trial. Scand J Surg. 2019;1457496919883812.

23. Groen VA, van de Graaf VA, Scholtes VA, Sprague S, van Wagensveld BA, Poolman RW. Effects of bariatric surgery for knee complaints in (morbidly) obese adult patients: a systematic review. Obes Rev. 2015;16:161-70.

24. Parvizi J, Trousdale RT, Sarr MG. Total joint arthroplasty in patients surgically treated for morbid obesity. J Arthroplasty. 2000;15:1003-8.

Publisher's Note Springer Nature remains neutral with regard to jurisdictional claims in published maps and institutional affiliations. 